\title{
GLOBAL SOLVABILITY OF A DISSIPATIVE FRÉMOND MODEL FOR SHAPE MEMORY ALLOYS. PART I: MATHEMATICAL FORMULATION AND UNIQUENESS
}

\author{
BY
}

\author{
ELENA BONETTI
}

\begin{abstract}
Dipartimento di Matematica "F. Casorati", Università di Pavia, Via Ferrata 1, 27100 Pavia, Italy
\end{abstract}
\begin{abstract}
The mathematical formulation of a dissipative Frémond model for shape memory alloys is given in terms of an initial and boundary values problem. Uniqueness of sufficiently regular solutions is proved by use of a contracting estimates procedure in the case when quadratic dissipative contributions are neglected in the energy balance. The related existence result is only established while its proof will be detailed by the author in a subsequent paper.
\end{abstract}

1. Introduction. This paper deals with a macroscopic thermomechanical model describing the solid-solid martensitic transformation in shape memory alloys (SMA). Shape memory materials are characterized by the fact that they can be permanently deformed under mechanical stresses and then recover their original shape just by thermal means. The striking and well known properties of shape memory alloys result from these links between mechanical and thermal actions (cf. [26, 32]). This phenomenon can be ascribed to a structural phase transition between two different configurations of the metallic lattice, one symmetric phase appearing at high temperature, called austenite, and one twinned organized phase prevailing at low temperature, called martensite (cf. [30, 16, 29, 1]). Let us note in advance that even if in the realistic situations 24 different crystallographically equivalent variants of martensite are present, being concerned with a macroscopic description of the thermomechanical behavior of SMA, and in agreement with the different symmetries of the two phases, the model we are dealing with considers only one variant of austenite and two variants of martensite and it is assumed that they may coexist at each point (cf. [22]). Nonetheless, we point out that this approach is sufficient to provide a consistent and exhaustive description of the SMA peculiar behavior. We also recall that SMA stress-strain relations are strongly dependent on temperature and they include

Received December 27, 2001.

2000 Mathematics Subject Classification. Primary 35K85, 74Nxx, 35Q72.

E-mail address: bonetti@dimat.unipv.it 
hysteretic branches (cf. $[17,21,34])$. Indeed, the shape memory effect results from temperature dependence of hysteresis loops, as the thermal and mechanical actions modify the phase composition of the material.

We aim to investigate a model proposed by Frémond which describes the shape memory effect in terms of thermodynamics and continuum mechanics laws and accounting for micromechanics in order to achieve the estimate of the interaction energy due to phase transition. In addition, this model, which can refer to any dimension of space, forces the internal quantities to be submitted to mechanical constraints in order to guarantee the physical consistence of the phase variables and it includes microscopic movements and diffusive effects in the power of interior forces. In past years, several papers have investigated the Frémond model (cf. $[17,18])$ from a mathematical point of view, but only for some approximated version (cf. [11]). Indeed, in these papers the corresponding PDE's system has been written by neglecting dissipative effects and microscopic movements in the power of interior forces. In particular, some viscosity terms were added to provide a good description of the evolution of the phase parameters. Moreover, in a first instance, only diffusionless martensitic phase transitions have been considered. Our approach is rather different, as we aim to derive the complete dissipative model accounting for microscopic velocities and diffusive phase transformations (cf. [22, 21]). We will detail in the next section how the model is derived in terms of two functionals, the free energy and the pseudo-potential of dissipation. Now, for the sake of completeness, we aim to present some results related to the above approximated version of the Fremond model. The associated initial boundary values problem is written in terms of the temperature (Kelvin), the small deformations, and the phase proportions of austenite and martensite. In this framework, the model is recovered by the free energy functional, which is given by the sum of the specific free energies of the phases and an interaction energy term (cf. [11]). Constitutive equations, along with the second principle of thermodynamics and the universal conservation laws for energy and momentum, lead to an evolution problem for three partial differential equations, completed by suitable initial and boundary conditions. In addition, we point out that, since the model by Frémond accounts for mechanically induced phase transformations, mechanical actions are described by means of the second gradient theory (cf. [25]), even if a simpler setting is considered by restricting to diagonal components of the strain tensor. Nevertheless, this formulation supplies a useful fourth-order term in the momentum balance. Yet in this simplified version, the resulting system is highly nonlinear, especially due to three terms in the energy balance equation coupling displacements, temperature, and phase fractions, whereas such equation has a parabolic character whenever one assumes the standard Fourier law for the heat flux. Concerning this first approximated form of the model, we now present some related works. An existence and uniqueness result has been proved in [11] for a first version of the resulting initial boundary values problem, in the case when all the nonlinearities in the balance of the energy are neglected and the momentum balance equation is assumed in the quasi-stationary form. Next, let us briefly review some results concerning the solvability of the problem in which nonlinear terms are retained in the energy balance. We now refer to this model as the complete one, even if the approach we are going to introduce in the remainder of the paper will extend this setting by accounting 
for dissipative effects and microscopic forces in the power of interior forces and by retaining some diffusion terms for the phase dynamics. The full one-dimensional model turns out to be well-posed both in the quasi-stationary case as in the more delicate case of a hyperbolic momentum equation. For the first result see [13], while the existence proof for the latter has been presented in two independent papers [14] and [33]. Moreover, a related uniqueness result is given in [8]. Finally, we point out that the full quasi-static three-dimensional model admits a unique solution under suitable regularity and compatibility assumptions on the thermal expansion coefficient, which are verified by realistic data (cf. [10] and [8]). Concerning the thermodynamical coherence of the above model, we recall that it is based on the positivity assumption on the temperature. In [12], under rather weak assumptions on the data of the system, it is proved that any sufficiently smooth solution of the governing field equations has, indeed, the property that the absolute temperature variable attains positive values almost everywhere. We finally recall a recent paper (cf. [6]) in which we have investigated the Frémond model in the case when a diffusive phase transformation and dissipative thermal effects are assumed, i.e., the heat flux law is specified in the framework of the Gurtin-Pipkin theory.

The problem we aim to investigate in this paper is rather different since we present the complete three dimensional dissipative model proposed by Frémond for which, to our knowledge, no analytical results have been obtained. Hence, we derive a related initial and boundary values problem that we study from the point of view of existence and uniqueness of a solution. In particular, in this paper we can state uniqueness of sufficiently regular solutions of the corresponding PDE's system in the case when we neglect higher order dissipative terms in the energy balance. Next, the existence of a solution fulfilling the required regularity will be presented in a subsequent related paper, even in the case when one retains some quadratic dissipative terms in the actual power of interior forces.

2. The dissipative model. In this section we derive the initial and boundary values problem which is related to the complete macroscopic thermomechanical model proposed by Frémond (cf. $[22,21]$ ) to describe shape memory behavior and including dissipative effects and microscopic velocities in the constitutive equations as well as microscopic forces in the principle of virtual power. In the above physical framework, the internal constraints on the state quantities will play an important role and account for most of the striking properties of the shape memory materials, so that they will be included in the free energy functional.

We take a sample of SMA located in a bounded smooth domain $\Omega \subset \mathbf{R}^{3}$ and consider the thermomechanical evolution of this system, during a finite time interval $[0, T]$. We write the model in terms of the absolute temperature $\theta$, the strain tensor $\varepsilon(\mathbf{u})$ ( $\mathbf{u}$ being the vector of small displacements), and the volumetric fractions of the variant of austenite $\beta_{3}$ and the two variants of martensite $\left(\beta_{1}, \beta_{2}\right)$. In order to achieve a precise description of the phenomenon, we have to take into account both the equilibrium and the evolution of the system, which are described by two functionals: the free energy $\Psi$, defined on the state variables, and the pseudo-potential of dissipation $\Phi$, defined on dissipative variables. 
It is known that the state of equilibrium of a thermomechanical system is characterized by the fact that the state variables do not vary in time. Thus it is straightforward that this notion depends on the choice of the set $E$ of the state variables. We make precise $E$ as follows:

$$
E:=\left\{\theta, \varepsilon(\mathbf{u}), \nabla \operatorname{tr} \varepsilon(\mathbf{u}), \beta_{1}, \beta_{2}, \beta_{3}, \nabla \beta_{1}, \nabla \beta_{2}, \nabla \beta_{3}\right\} .
$$

With regard to the choice of $E$, let us point out that the presence of the gradients of the phases $\nabla \beta_{i}$ corresponds to assume that the microstructure of the material at one point is influenced by its neighborhood. Analogously, due to the presence of the diffusive term $\nabla \operatorname{tr} \varepsilon(\mathbf{u})$, which on the contrary is usually neglected in the first gradient theory, we are accounting for mechanical actions exerted on surfaces, but we restrict to a simple formulation involving just the diagonal components of the strain tensor. Hence, we introduce the free-energy functional $\Psi$ defined on $E$. Due to the composition of SMA, we write the free energy as the sum of the specific free energies of the phases $\left(\psi_{i}, i=1,2,3\right.$, where the indexes correspond to that of volume fractions), weighted with their proportions $\beta_{i}$, and an interaction energy term $I e$ (see e.g. [5, 23] for other examples of mixture free energy). For the sake of simplicity, in the sequel we assume the material to be isotropic and fix its density as $\rho=1$, so that the free energy can be written as follows:

$$
\Psi(E)=\sum_{i=1}^{3} \beta_{i} \psi_{i}+I e .
$$

Let us first address the specific free energies of the austenite and the two martensite variants,

$$
\begin{aligned}
& \psi_{1}=\frac{1}{2} \varepsilon R \varepsilon+\frac{\nu}{2}|\nabla \operatorname{tr} \varepsilon|^{2}-\alpha(\theta) \operatorname{tr} \varepsilon-c_{s} \theta \log \theta, \\
& \psi_{2}=\frac{1}{2} \varepsilon R \varepsilon+\frac{\nu}{2}|\nabla \operatorname{tr} \varepsilon|^{2}+\alpha(\theta) \operatorname{tr} \varepsilon-c_{s} \theta \log \theta, \\
& \psi_{3}=\frac{1}{2} \varepsilon R \varepsilon+\frac{\nu}{2}|\nabla \operatorname{tr} \varepsilon|^{2}-\lambda(\theta)-c_{s} \theta \log \theta,
\end{aligned}
$$

where $R$ denotes the elastic tensor, $c_{s}$ the heat capacity of the phase transition, $\nu$ is a positive constant, and $\alpha$ and $\lambda$ two real functions of the temperature, whose properties will be specified in a moment, the first accounting for the thermal expansion energy and the latter for the energy associated to the phase transition. The function $\alpha$ is assumed to be nonincreasing and positive, but vanishing over a critical temperature $\theta_{c}$ (the socalled Curie temperature), while $\lambda$ is nondecreasing and it vanishes for the transition temperature $\theta_{*}<\theta_{c}$. In the original Frémond model $\lambda$ was an affine function of the absolute temperature. Let us observe that the assumptions on $\alpha$ correspond to the fact that at high temperatures SMA present mainly an elastic behavior (cf. [31]). We also recall that, as it is usually in elasticity theory, the following relation holds (cf. [9]):

$$
R \varepsilon(\mathbf{u})=\lambda_{L} \operatorname{tr} \varepsilon \mathbf{1}+2 \mu_{L} \varepsilon(\mathbf{u}),
$$

where $\lambda_{L}$ and $\mu_{L}$ stand for the Lamé constants and 1 for the identity matrix.

Next, we have to specify the interaction energy term Ie in (2.2) in which, in particular, we include internal mechanical constraints forcing the phases to attain only meaningful 
values, in the sense that no void or overlapping can occur. To this aim, we prescribe

$$
0 \leq \beta_{i} \leq 1, i=1,2,3 \text { and } \sum_{i=1}^{3} \beta_{i}=1 .
$$

Note that, by (2.7), we could write the model only in terms of the martensite phase proportions, as $\beta_{3}$ can be derived by the relation

$$
\beta_{3}=1-\beta_{1}-\beta_{2} \text {. }
$$

In terms of the only martensitic variants, $(2.7)$ is equivalent to require that $\left(\beta_{1}, \beta_{2}\right)$ belong to the convex of $\mathbf{R}^{2}$ :

$$
C:=\left\{\left(\gamma_{1}, \gamma_{2}\right) \in \mathbf{R}^{2}: 0 \leq \beta_{i} \leq 1 \text { and } \sum_{i=1}^{2} \beta_{i} \leq 1\right\} .
$$

Thus, in the following we restrict the set $E$ of the state variables (cf. (2.1)) by including only the phase parameters $\left(\beta_{1}, \beta_{2}\right)$. Hence, by considering (2.7) as a material property, it is written in $\Psi$, and more precisely in the interaction energy term $I e$, as it involves both the phases. We proceed by extending $\Psi$ to be defined for any values of the phase proportions, including those that are physically impossible (i.e., $\left(\beta_{1}, \beta_{2}\right) \notin C$ ), but we assign the value $+\infty$ in correspondence to these cases. To this aim, we introduce the indicator function $I_{C}$ of the convex $C$, which is defined by:

$$
I_{C}\left(\gamma_{1}, \gamma_{2}\right)=0 \text { if }\left(\gamma_{1}, \gamma_{2}\right) \in C \text { and } I_{C}\left(\gamma_{1}, \gamma_{2}\right)=+\infty \text { otherwise. }
$$

In agreement with the above position, the interaction term can also be written only in terms of the martensite variants, as it is straightforward to deduce by (2.8) that $\nabla \beta_{3}=-\nabla\left(\beta_{1}+\beta_{2}\right)$. We globally write (cf., i.e., $\left.[22,21]\right)$

$$
I e=\frac{\eta}{2} \sum_{i=1}^{2}\left|\nabla \beta_{i}\right|^{2}+I_{C}\left(\beta_{1}, \beta_{2}\right),
$$

$\eta$ being a nonnegative diffusive parameter. Note that, in the case $\eta=0$, we recover a diffusion free martensite phase transition model, in which the gradients of the phases are not actually included in the set $E$ of the state variables (cf., i.e., [11]).

Thus, by the free energy, constitutive equations can be derived for the nondissipative physical quantities, while dissipation of the shape memory behavior is concerned with the variation of internal quantities, i.e., the phase proportions of austenite and martensite. We include dissipation in the model by following the approach proposed by Moreau (cf. [28]) and introduce the pseudo-potential of dissipation $\Phi$ as a real function characterized by the fact that

$$
\begin{aligned}
& \Phi(0)=0, \\
& \Phi \geq 0, \\
& \Phi \text { is convex with respect to the dissipative variables. }
\end{aligned}
$$

To make precise the set of the dissipative variables $\delta E$, we first observe that (cf. (2.8)) $\beta_{3 t}=-\left(\beta_{1 t}+\beta_{2 t}\right)$. Thus, in a general situation, we fix

$$
\delta E:=\left\{\beta_{1 t}, \beta_{2 t}, \nabla \beta_{1 t}, \nabla \beta_{2 t}\right\},
$$


and

$$
\Phi(\delta E):=\frac{\zeta}{2} \sum_{i=1}^{2}\left(\beta_{i t}\right)^{2}+\frac{\delta}{2} \sum_{i=1}^{2}\left|\nabla \beta_{i t}\right|^{2},
$$

$\zeta$ and $\delta$ being nonnegative parameters.

Hence, a PDE's system describing the thermomechanical model can be written, in accordance with the second principle of thermodynamics (cf. [24]), in terms of the free energy and the pseudo-potential of dissipation owing to the universal balance laws, i.e., the energy balance and the principle of virtual power. The main feature of Frémond's model is given by the inclusion of microscopic forces and dissipative effects in the principle of virtual power. Thus, accounting also for microscopic forces, the energy balance is specified by (for the notation used for the products we refer to [22])

$$
e_{t}+\operatorname{div} \mathbf{q}=r+\sigma \varepsilon_{t}+\mathbf{M} \cdot \nabla \operatorname{tr} \varepsilon_{t}+B \cdot \beta_{t}+\mathbf{H} \cdot \nabla \beta_{t},
$$

where $\beta$ stands for the vector with components $\left(\beta_{1}, \beta_{2}\right)$ and some of the above quantities include dissipative effects. Now, we aim to make clear the physical meaning of the ingredients of (2.14). By $e$ we denote the internal energy, by $\mathbf{q}$ the heat flux vector, for which we will state later a suitable boundary condition, and the right hand side of the above relation includes the actual power of interior forces. More precisely, $r$ is a rate of heat production, the terms involving the stress tensor $\sigma$ and the vector of forces $\mathbf{M}$ correspond to macroscopic mechanically induced heat sources, while heat sources induced by microscopic forces are collected by the last two terms involving the vectors $B$ and $\mathbf{H}$, whose physical meaning will follow by the principle of virtual power written for microscopic movements as well as boundary condition prescribed on $\mathbf{H}$.

Next, we write explicitly the principle of virtual power. We restrict our analysis to the case when the acceleration forces are zero, so that denoting by $P_{i}$ the power of interior forces and by $P_{e}$ the power of exterior forces, the principle of virtual power turns out to be written as (cf. $[24,21,20])$

$$
P_{i}+P_{e}=0
$$

where $P_{i}$ and $P_{e}$ will be specified in a moment.

For any domain $D \subseteq \Omega$ and for any virtual macroscopic velocity $\mathbf{v}$ and microscopic velocity $c$, as virtual power of interior forces we set (cf. [22])

$$
P_{i}(D, \mathbf{v}, c)=-\int_{D} \sigma \varepsilon(\mathbf{v})-\int_{D} \mathbf{M} \cdot \nabla \operatorname{tr} \varepsilon(\mathbf{v})-\int_{D} B c-\int_{D} \mathbf{H} \cdot \nabla c
$$

and for the exterior

$$
P_{e}(D, \mathbf{v}, c)=\int_{D} \mathbf{G} \cdot \mathbf{v}+\int_{\partial D} \mathbf{g} \cdot \mathbf{v}+\int_{D} A c+\int_{\partial D} a c,
$$

where $\mathbf{G}$ and $\mathbf{g}$ stand for volumic and surfacic, respectively, force vectors applied to the body and involving macroscopic displacements, while $A$ and $a$ denote the amount of volumic and surfacic mechanical energy provided on the domain by external actions involving only microscopic movements. However, for the sake of simplicity, we will put $A=a=0$. Thus, if we let $c=0$ in (2.16) and (2.17), from (2.15) we can recover the classical quasi-static equilibrium equation

$$
\operatorname{div}(-(\operatorname{div} \mathbf{M}) \mathbf{1}+\sigma)+\mathbf{G}=0,
$$


and then specify associated suitable boundary conditions. In particular, letting $\partial \Omega=$ $\Gamma=\Gamma_{0} \cup \Gamma_{1}$, we prescribe that

$$
\begin{aligned}
& \mathbf{u}=0 \text { on } \Gamma_{0} \times(0, T), \\
& (-(\operatorname{div} \mathbf{M}) \mathbf{1}+\sigma) \cdot \mathbf{n}=\mathbf{g} \text { on } \Gamma_{1} \times(0, T), \\
& \mathbf{M} \cdot \mathbf{n}=0 \text { on } \Gamma \times(0, T),
\end{aligned}
$$

where $\mathbf{n}$ stands for the normal unit vector to the boundary $\Gamma$. We point out that $(2.20)$ corresponds to assume that a known traction $\mathbf{g}$ acts on a part of the boundary, while the body is fixed on the other one by (2.19). Hence, (2.21) means that no double forces occur on the surface.

Analogously, we can get $\mathbf{v}=0$ in (2.16)-(2.17) and recover from (2.15) the principle of virtual power written for microscopic movements,

$$
B-\operatorname{div} \mathbf{H}=0,
$$

coupled with the boundary condition

$$
\mathbf{H} \cdot \mathbf{n}=0 \text { on } \Gamma \times(0, T),
$$

from which we can deduce the mechanical meaning of the vector $\mathbf{H}$, which indicates a work flux vector, while $B$ is a vector collecting microscopic forces.

Now, it remains to state the constitutive equations of the model and substitute them in the balance laws (2.14), (2.18), and (2.22). We first recall that the internal energy $e$ is related to the entropy $s$, as it is usual, by

$$
e=\Psi+\theta s
$$

$s$ being specified by the Helmotz relation

$$
s=-\frac{\partial \Psi}{\partial \theta} .
$$

As we do not consider dissipation for the macroscopic stresses, we state

$$
\begin{aligned}
& \sigma=\frac{\partial \Psi}{\partial \varepsilon}, \\
& \mathbf{M}=\frac{\partial \Psi}{\partial \nabla \operatorname{tr} \varepsilon} .
\end{aligned}
$$

On the contrary, we include dissipative effects both in $B$ and in $\mathbf{H}$ (cf. (2.12)-(2.13)) and prescribe that they are given by the sum of a dissipative and a non-dissipative contribution. Precisely, we specify $B$ as

$$
B=B^{n d}+B^{d} \in \frac{\partial \Psi}{\partial \beta}+\frac{\partial \Phi}{\partial \beta_{t}},
$$

and $\mathbf{H}$ as

$$
\mathbf{H}=\mathbf{H}^{n d}+\mathbf{H}^{d} \in \frac{\partial \Psi}{\partial \nabla \beta}+\frac{\partial \Phi}{\partial \nabla \beta_{t}} .
$$

Let us note that by abuse of notation (cf. [22]), we have denoted $B$ and $\mathbf{H}$ as a scalar and a vector quantity, respectively, while dealing with two phase variables $B$ is actually 
a vector and $\mathbf{H}$ a matrix. Finally, the heat flux vector $\mathbf{q}$ in (2.14) is assumed to fulfill the standard Fourier law

$$
\mathbf{q}(x, t)=-k_{0} \nabla \theta(x, t), \quad(x, t) \in \Omega \times(0, T),
$$

with $k_{0}>0$, and coupled with Neumann boundary condition

$$
-\mathbf{q} \cdot \mathbf{n}=h \text { in } \Gamma \times(0, T),
$$

which corresponds to prescribe a known heat flux $h$ through the boundary.

In order to show that the model we have just introduced satisfies the second principle of thermodynamics, i.e., the Clausius-Duhem inequality (cf. [19]), it is more convenient to follow the approach by Frémond and introduce formally the heat flux vector $\mathbf{q}$ as a dissipative quantity defined by $\Phi$. Thus, we let $\nabla \theta$ belong to $\delta E$ and $\Phi$ depend also on the absolute temperature (cf. $[22,21])$, i.e.,

$$
\delta E:=\left\{\beta_{1 t}, \beta_{2 t}, \nabla \beta_{1 t}, \nabla \beta_{2 t}, \nabla \theta\right\},
$$

and

$$
\Phi(\delta E, \theta)=\frac{\zeta}{2} \sum_{i=1}^{2}\left(\beta_{i t}\right)^{2}+\frac{\delta}{2} \sum_{i=1}^{2}\left|\nabla \beta_{i t}\right|^{2}+\frac{k_{0}}{2 \theta}|\nabla \theta|^{2},
$$

for $k_{0}>0$. Hence, we introduce a new dissipative quantity

$$
\mathbf{Q}^{d}=-\frac{\partial \Phi}{\partial \nabla \theta}=-\frac{k_{0}}{\theta} \nabla \theta
$$

so that letting

$$
\mathbf{q}=\theta \mathbf{Q}^{d}
$$

yields the Fourier relation (2.30). Then, if one substitutes (2.25)-(2.29) and (2.34)-(2.35) in (2.14), by the chain rule, some terms cancel and we can equivalently write the energy balance as follows:

$$
\theta s_{t}+\theta \operatorname{div} \mathbf{Q}^{d}-R \theta=-\mathbf{Q}^{d} \cdot \nabla \theta+B^{d} \cdot \beta_{t}+\mathbf{H}^{d} \cdot \nabla \beta_{t},
$$

where (recall that $\theta$ is the absolute temperature) $R=r / \theta$. Thus, after observing that by $(2.28),(2.29)$, and (2.34) the right hand side of $(2.36)$ corresponds to

$$
\left(\frac{\partial \Phi}{\partial \nabla \theta}, \frac{\partial \Phi}{\partial \beta_{t}}, \frac{\partial \Phi}{\partial \nabla \beta_{t}}\right) \cdot\left(\nabla \theta, \beta_{t}, \nabla \beta_{t}\right),
$$

we get (cf. (2.32))

$$
-\mathbf{Q}^{d} \cdot \nabla \theta+B^{d} \cdot \beta_{t}+\mathbf{H}^{d} \cdot \nabla \beta_{t}=\mathbf{F}^{d} \cdot \delta E \geq 0
$$

where

$$
\mathbf{F}^{d}=\left(\frac{\partial \Phi}{\partial \nabla \theta}, \frac{\partial \Phi}{\partial \beta_{t}}, \frac{\partial \Phi}{\partial \nabla \beta_{t}}\right) \in \partial \Phi(\delta E),
$$

$\partial \Phi$ denoting the subdifferential of $\Phi$ with respect to the dissipative variables. Indeed, as (2.11) holds, the subdifferential of $\Phi$ turns out to be a maximal monotone operator and (2.38) follows by standard monotonicity arguments and the fact that $0 \in \partial \Phi(0)$ (cf. (2.11) and (2.39)). Finally, dividing (2.36) by the absolute temperature $\theta$ yields the second principle of thermodynamics, namely (cf. (2.38))

$$
s_{t}+\operatorname{div} \mathbf{Q}^{d}-R=\frac{1}{\theta} \mathbf{F}^{d} \cdot \delta E \geq 0 .
$$


Now, we can write the system of PDE's in terms of the unknowns, by substituting in (2.14) (or in its equivalent version (2.36)), (2.18), and (2.22) the constitutive equations of the model specified by the free energy and the pseudo-potential of dissipation. In particular, by (2.25) (recall (2.2)-(2.5) and (2.10)), we deduce

$$
s=c_{s}(1+\log \theta)-\alpha^{\prime}(\theta)\left(\beta_{2}-\beta_{1}\right) \operatorname{tr} \varepsilon(\mathbf{u})+\lambda^{\prime}(\theta)\left(1-\left(\beta_{1}+\beta_{2}\right)\right),
$$

and, by $(2.26)-(2.27)$ and $(2.6)$,

$$
\begin{aligned}
& \sigma=\lambda_{L}(\operatorname{tr} \varepsilon(\mathbf{u})) \mathbf{1}+2 \mu_{L} \varepsilon(\mathbf{u})+\alpha(\theta)\left(\beta_{2}-\beta_{1}\right) \mathbf{1}, \\
& M=\nu \nabla \operatorname{tr} \varepsilon(\mathbf{u}) .
\end{aligned}
$$

Finally, we write the vectors of microscopic forces (2.28) and (2.29) on account of the free energy and (2.13):

$$
\begin{aligned}
& B=\left(\begin{array}{l}
\lambda(\theta)-\alpha(\theta) \operatorname{tr} \varepsilon(\mathbf{u}) \\
\lambda(\theta)+\alpha(\theta) \operatorname{tr} \varepsilon(\mathbf{u})
\end{array}\right)+\partial I_{C}\left(\beta_{1}, \beta_{2}\right)+\zeta\left(\begin{array}{c}
\beta_{1 t} \\
\beta_{2 t}
\end{array}\right), \\
& \mathbf{H}=\eta\left(\begin{array}{l}
\nabla \beta_{1} \\
\nabla \beta_{2}
\end{array}\right)+\delta\left(\begin{array}{c}
\nabla \beta_{1 t} \\
\nabla \beta_{2 t}
\end{array}\right) .
\end{aligned}
$$

In particular, let us point out that $\partial I_{C}$ in (2.44) stands for the subdifferential of the indicator function of the convex $C$ and it is obtained as a generalized derivative with respect to $\left(\beta_{1}, \beta_{2}\right)$ of the nonsmooth function $I_{C}$ in $(2.10)$. From a mechanical point of view, it represents a thermodynamical reaction accounting for the internal constraint (2.7). Indeed, $\partial I_{C}\left(\beta_{1}, \beta_{2}\right)$ is not an empty set if and only if $\left(\beta_{1}, \beta_{2}\right) \in C$, which ensures that (2.7) holds (cf. (2.9)). Moreover, the thermodynamical reaction $\partial I_{C}\left(\beta_{1}, \beta_{2}\right)$ is zero if $\left(\beta_{1}, \beta_{2}\right)$ belongs to the interior of $C$, while a normal reaction force appears in $(2.44)$ if $\left(\beta_{1}, \beta_{2}\right)$ lies on the boundary of $C$, as $\partial I_{C}\left(\beta_{1}, \beta_{2}\right)$ coincides with the cone of normal vectors to the boundary at the point $\left(\beta_{1}, \beta_{2}\right)$.

Now, recalling also $(2.30)$, by virtue of $(2.41)-(2.45)$, we are in the position of deducing the complete PDE's system originating from (2.36) (cf. (2.14), (2.18), and (2.22)):

$$
\begin{gathered}
\left(c_{s}-\theta \alpha^{\prime \prime}(\theta) \operatorname{tr} \varepsilon\left(\beta_{2}-\beta_{1}\right)+\theta \lambda^{\prime \prime}(\theta)\left(1-\left(\beta_{1}+\beta_{2}\right)\right)\right) \theta_{t}-k_{0} \Delta \theta \\
=r+\theta \alpha^{\prime}(\theta)\left(\beta_{2}-\beta_{1}\right) \operatorname{tr} \varepsilon_{t}+\theta \alpha^{\prime}(\theta) \operatorname{tr} \varepsilon\left(\beta_{2}-\beta_{1}\right)_{t}+\theta \lambda^{\prime}(\theta)\left(\beta_{1}+\beta_{2}\right)_{t} \\
+\zeta \sum_{i=1}^{2}\left(\beta_{i t}\right)^{2}+\delta \sum_{i=1}^{2}\left|\nabla \beta_{i t}\right|^{2} \\
\zeta\left(\begin{array}{c}
\beta_{1 t} \\
\beta_{2 t}
\end{array}\right)-\delta\left(\begin{array}{c}
\Delta \beta_{1 t} \\
\Delta \beta_{2 t}
\end{array}\right)-\eta\left(\begin{array}{c}
\Delta \beta_{1} \\
\Delta \beta_{2}
\end{array}\right)+\partial I_{C}\left(\beta_{1}, \beta_{2}\right) \\
+\left(\begin{array}{c}
-\alpha(\theta) \operatorname{tr} \varepsilon+\lambda(\theta) \\
\alpha(\theta) \operatorname{tr} \varepsilon+\lambda(\theta)
\end{array}\right) \ni\left(\begin{array}{l}
0 \\
0
\end{array}\right), \\
\operatorname{div}\left(-\nu \Delta \operatorname{tr} \varepsilon(\mathbf{u}) \mathbf{1}+\lambda_{L} \operatorname{tr} \varepsilon(\mathbf{u}) \mathbf{1}+2 \mu_{L} \varepsilon(\mathbf{u})+\alpha(\theta)\left(\beta_{2}-\beta_{1}\right) \mathbf{1}\right)+\mathbf{G}=\mathbf{0} .
\end{gathered}
$$

Actually, we deal with a slightly modified version of the above system, as we consider $\zeta=\delta=0$ in $(2.46)$. From a mechanical point of view, this corresponds to require that dissipative quadratic effects on the phases are negligible in the power of interior forces with respect to the other mechanically induced heat sources, which is reasonable in the 
framework of small perturbations assumption (we refer to [24] for a detailed presentation of this subject). However, let us point out in advance that in a subsequent paper we will prove an existence result for a system obtained by $(2.46)-(2.48)$ in the case when $\delta=0$ in $(2.46)$ but $\zeta \neq 0$, i.e., we do not neglect quadratic dissipative contributions of the phases but only of their gradients in the actual power of interior forces.

REMARK 2.1. Let us point out that the gradients of the phase proportions have been introduced in the model to provide a more sophisticated description of the microscopic phase transition phenomenon. Indeed, the presence of the gradients accounts for local structural interactions between the phases (cf., e.g., [22, p. 17]). This position has been fully justified in the framework of thermomechanical macroscopic modelling for different phase transitions phenomena described via continuum mechanics laws (the reader can refer, e.g., to [21] and references therein for a detailed argumentation, also in relation with experimental results).

REMARK 2.2. Let us briefly discuss the fact that we neglect in (2.46) higher order dissipative contributions, while we retain the corresponding terms in (2.47). After assuming the small perturbations assumption, our position turns out to be reasonable from a mechanical point of view, once recalling that even if one assumes that the mechanically induced heat sources by microscopic movements or their gradients are negligible in the energy balance (in which we have also macroscopic contributions), the same terms are relevant in the description of phases dynamics, which are obviously related to microscopic velocities. From a mathematical point of view, these dissipative contributions seem to be necessary to get some global solvability of the system and uniqueness of the solutions.

In this concern and in relation with the results in the literature on the Frémond model, let us observe that we have introduced the mathematical derivation of the complete model, i.e., writing the principle of virtual power in a generalized form including microscopic forces, which is, to our knowledge, a new modeling approach. Moreover, under suitable thermomechanical assumptions, we investigate two slightly approximated versions, in which only dissipative quadratic terms are neglected in the energy balance. In addition, let us point out that we are able to state a global existence result in the case when we retain some of these quadratic higher order nonlinearities.

Then, we complete the previous statement by suitable Cauchy conditions for the temperature and the phases

$$
\begin{gathered}
\theta(0)=\theta_{0}, \\
\beta_{i}(0)=\beta_{i 0}, \quad i=1,2,
\end{gathered}
$$

and natural boundary assumptions derived from (2.19)-(2.21) and (2.23)

$$
\begin{aligned}
& \mathbf{u}=0 \text { on } \Gamma_{0} \times(0, T), \\
& \left(\left(-\nu \Delta \operatorname{tr} \varepsilon(\mathbf{u})+\lambda_{L} \operatorname{tr} \varepsilon(\mathbf{u})+\alpha(\theta)\left(\beta_{2}-\beta_{1}\right)\right) \mathbf{1}+2 \mu_{L} \varepsilon(\mathbf{u})\right) \cdot \mathbf{n}=\mathbf{g} \\
& \text { on } \Gamma_{1} \times(0, T), \\
& \partial_{n} \operatorname{tr} \varepsilon(\mathbf{u})=0 \text { on } \Gamma \times(0, T), \\
& \partial_{n} \beta_{i t}=\partial_{n} \beta_{i}=0, \quad i=1,2 \text { on } \Gamma \times(0, T) .
\end{aligned}
$$


In addition, by virtue of (2.30), we can write (2.31) as follows

$$
k_{0} \partial_{n} \theta=h \text { on } \Gamma \times(0, T) .
$$

3. Abstract formulation and main results. For the sake of simplicity, we write the system (2.46)-(2.48) in terms of two new phase variables (cf. [11])

$$
\chi_{1}:=\beta_{1}+\beta_{2} \text { and } \chi_{2}:=\beta_{2}-\beta_{1},
$$

and, to simplify notations, we substitute $\zeta$ and $\delta$ in (2.13) and $\eta$ in (2.10) by $2 \zeta, 2 \delta$, and $2 \eta$, respectively. Hence, recalling $(2.9)$, we construct a new convex $\widetilde{K}$

$$
\widetilde{K}:=\left\{\left(\gamma_{1}, \gamma_{2}\right) \in \mathbf{R}^{2}:\left|\chi_{2}\right| \leq \chi_{1} \leq 1\right\},
$$

obviously obtained by $C$ in view of (3.1). We also recall that $\varepsilon(\mathbf{u})$ denotes the usual linearized strain tensor, i.e., $\varepsilon_{i j}(\mathbf{v})=\frac{1}{2}\left(\partial_{x_{i}} v_{j}+\partial_{x_{j}} v_{i}\right)$, so that it is straightforward $\operatorname{tr} \varepsilon(\mathbf{u})=\operatorname{div} \mathbf{u}$. Thus, the system (2.46)-(2.48) can be rewritten as follows (recall that we take $\zeta=\delta=0$ in $(2.46))$ :

$$
\begin{aligned}
& \left(c_{s}-\theta \alpha^{\prime \prime}(\theta) \chi_{2} \operatorname{div} \mathbf{u}+\theta \lambda^{\prime \prime}(\theta)\left(1-\chi_{1}\right)\right) \theta_{t}-k_{0} \Delta \theta=r+\theta \alpha^{\prime}(\theta) \chi_{2} \operatorname{div} \mathbf{u}_{t} \\
& +\theta \alpha^{\prime}(\theta) \operatorname{div} \mathbf{u} \chi_{2 t}+\theta \lambda^{\prime}(\theta) \chi_{1 t} \\
& \zeta\left(\begin{array}{c}
\chi_{1 t} \\
\chi_{2 t}
\end{array}\right)-\delta\left(\begin{array}{c}
\Delta \chi_{1 t} \\
\Delta \chi_{2 t}
\end{array}\right)-\eta\left(\begin{array}{c}
\Delta \chi_{1} \\
\Delta \chi_{2}
\end{array}\right)+\frac{1}{2} \partial I_{\widetilde{K}}\left(\chi_{1}, \chi_{2}\right) \ni\left(\begin{array}{c}
-\lambda(\theta) \\
-\alpha(\theta) \operatorname{div} \mathbf{u}
\end{array}\right), \\
& \operatorname{div}\left(\left(-\nu \Delta \operatorname{div} \mathbf{u}+\lambda_{L} \operatorname{div} \mathbf{u}+\alpha(\theta) \chi_{2}\right) \mathbf{1}+2 \mu_{L} \varepsilon(\mathbf{u})\right)+\mathbf{G}=\mathbf{0}
\end{aligned}
$$

and it is associated to the natural initial and boundary conditions derived from (2.49)(2.50) and (2.51)-(2.55). In particular, we substitute $(2.50)$ by

$$
\chi_{i}(0)=\chi_{i 0} \quad i=1,2
$$

where $\chi_{i 0}$ are, obviously, specified by (cf. (3.1))

$$
\chi_{10}=\beta_{10}+\beta_{20} \text { and } \chi_{20}=\beta_{20}-\beta_{10},
$$

and (2.54) by

$$
\partial_{n} \chi_{i t}=\partial_{n} \chi_{i}=0 \text { on } \Gamma \times(0, T), \quad i=1,2 .
$$

In addition, let us recall that the subdifferential $\partial I_{\widetilde{K}}$ is a maximal monotone operator in $\mathbf{R}^{2}$ defined as follows:

$$
\left(\begin{array}{l}
y_{1} \\
y_{2}
\end{array}\right) \in \partial I_{\widetilde{K}}\left(\chi_{1}, \chi_{2}\right) \text { if and only if }\left(\chi_{1}, \chi_{2}\right) \in \widetilde{K} \text { and } \sum_{i=1}^{2} y_{i}\left(x_{i}-\chi_{i}\right) \leq 0 \forall\left(x_{1}, x_{2}\right) \in \widetilde{K} \text {. }
$$

In particular, let us point out that $\frac{1}{2} \partial I_{\widetilde{K}}=\partial I_{\widetilde{K}}\left(\right.$ as $\left.\frac{1}{2}>0\right)$.

Our analysis refers to an abstract version of the above problem. Thus, we start by introducing the Hilbert triplet $V \hookrightarrow H \hookrightarrow V^{\prime}$ (cf. [27, p. 202]) with

$$
V:=H^{1}(\Omega) \text { and } H:=L^{2}(\Omega),
$$

where $H$ is identified as usual with its dual space $H^{\prime}$ and the above inclusions are continuous and compact. Next, we denote by $\langle\cdot, \cdot\rangle$ the duality pairing between $V^{\prime}$ and $V$ and, by abuse of notation, by $\|\cdot\|_{H}$ the norm both in $H$ and in $H^{N}$, with $N \geq 1$. 
Whereas the results we intend to present refer also to lower dimensional spaces (and sometimes they could be improved), we have restricted our investigation to the threedimensional framework as it turns out to be more meaningful from a physical point of view. Thus, let

$$
\Omega \subseteq \mathbf{R}^{3} \text { and } Q:=\Omega \times(0, T),
$$

where $T$ is a fixed final time. We assume that the domain $\Omega$ is smooth and such that its boundary $\Gamma$ is partitioned into two measurable sets $\Gamma_{0}$ and $\Gamma_{1}$, with the surface measure of $\Gamma_{0}$ being strictly positive. Hence, we consider a further Hilbert space $\mathbf{W}$, defined by

$$
\mathbf{W}:=\left\{\mathbf{v} \in V^{3}: \mathbf{v}=0 \text { on } \Gamma_{0} \text { and } \operatorname{div} \mathbf{v} \in V\right\},
$$

and endowed with the norm (cf. [10])

$$
\|\mathbf{v}\|_{\mathbf{W}}^{2}=\int_{\Omega}|\nabla \operatorname{div} \mathbf{v}|^{2}+\sum_{i, j=1}^{3} \int_{\Omega}\left(\partial_{x_{i}} v_{j}\right)^{2}, \quad \mathbf{v}=\left(v_{1}, v_{2}, v_{3}\right) \in \mathbf{W}
$$

In addition, let us introduce a bilinear continuous symmetric form on $\mathbf{W} \times \mathbf{W}$, specified by

$$
\begin{gathered}
a(\mathbf{w}, \mathbf{v})=\nu \int_{\Omega} \nabla \operatorname{div} \mathbf{w} \cdot \nabla \operatorname{div} \mathbf{v}+\lambda_{L} \int_{\Omega} \operatorname{div} \mathbf{w} \operatorname{div} \mathbf{v} \\
+2 \mu_{L} \sum_{i, j=1}^{3} \int_{\Omega} \varepsilon_{i j}(\mathbf{w}) \varepsilon_{i j}(\mathbf{v}) .
\end{gathered}
$$

By virtue of the Korn's inequality (cf. [15]), it is easy to deduce that $a$ is W-clliptic, in the sense that there exists a positive constant $C$ such that, for any $\mathbf{v} \in \mathbf{W}$, there holds

$$
a(\mathbf{v}, \mathbf{v}) \geq C\|\mathbf{v}\|_{\mathbf{W}}^{2} .
$$

On the other hand, it is a standard matter to check that (cf. [10])

$$
a(\mathbf{v}, \mathbf{v}) \geq \nu\|\nabla \operatorname{div} \mathbf{v}\|_{H}^{2}+\left(\lambda_{L}+2 \mu_{L} / 3\right)\|\operatorname{div} \mathbf{v}\|_{H}^{2},
$$

for any $\mathbf{v} \in \mathbf{W}$.

Hence, we introduce a convex subset of $H^{2}$ corresponding to $\widetilde{K}$ (cf. (3.2)). Let

$$
K:=\left\{\left(\gamma_{1}, \gamma_{2}\right) \in H^{2}:\left(\gamma_{1}, \gamma_{2}\right) \in \widetilde{K} \text { a.e. in } \Omega\right\},
$$

and note that there exists a positive constant $c_{K}$, depending only on $\widetilde{K}$, such that for any $\left(\gamma_{1}, \gamma_{2}\right) \in K$, there holds

$$
\left(\gamma_{1}(x)^{2}+\gamma_{2}(x)^{2}\right)^{1 / 2} \leq c_{K}
$$

for a.e. $x \in \Omega$. We point out that, whereas we can find an explicit bound for the phases as the form of $\widetilde{K}$ is known, we use the notation $c_{K}$ in (3.18) since we could extend our argumentation by considering instead of $\widetilde{K}$ any bounded convex subset of $\mathbf{R}^{2}$ (with $0 \in \widetilde{K})$. Finally, to put the problem (3.3)-(3.5) in the abstract setting of the above mentioned Hilbert spaces, we introduce the operators

$$
\begin{aligned}
& A: V \rightarrow V^{\prime}, \\
& \mathcal{H}: \mathbf{W} \rightarrow \mathbf{W}^{\prime} \\
& \mathcal{B}: H \rightarrow \mathbf{W}^{\prime},
\end{aligned}
$$


specified by

$$
\begin{aligned}
& \langle A u, v\rangle=\int_{\Omega} \nabla u \cdot \nabla v \quad u, v \in V, \\
& \mathbf{w}^{\prime}\langle\mathcal{H} \mathbf{w}, \mathbf{v}\rangle_{\mathbf{w}}=a(\mathbf{w}, \mathbf{v}) \quad \mathbf{w}, \mathbf{v} \in \mathbf{W}, \\
& \mathbf{w}^{\prime}\langle\mathcal{B} u, \mathbf{v}\rangle_{\mathbf{w}}=\int_{\Omega} u \operatorname{div} \mathbf{v} \quad u \in H, \mathbf{v} \in \mathbf{W} .
\end{aligned}
$$

Now, we set the hypotheses on the data prescribed in our analysis. Actually, in this paper we treat only the uniqueness of a solution for the initial and boundary values problem associated to the above model and thus we could avoid fixing regularity assumptions on the data of the problem, as it would be sufficient to prescribe some regularity of the solutions. Nonetheless, for the sake of completeness, we aim to derive the formulation of the problem with the regularity assumptions, we need to state the related existence result, and then prove uniqueness of the solution in this framework. Hence, concerning the Cauchy conditions (2.49) and (3.6), we assume

$$
\begin{aligned}
& \theta_{0} \in H^{1}(\Omega), \\
& \left(\chi_{10}, \chi_{20}\right) \in H^{2}(\Omega)^{2} \cap K, \quad \partial_{n} \chi_{i 0}=0 \quad i=1,2 \quad \text { on } \tilde{\mathbf{i}} .
\end{aligned}
$$

Then, we recall that the function $\alpha$ in (2.3) and (2.4) is a smooth nonnegative bounded function, nonincreasing, and vanishing over the interval $\left(\theta_{c},+\infty\right)$. More precisely, we let

$$
\begin{array}{ll}
\alpha \in C^{2}(\mathbf{R}), \quad\left\{\xi \in \mathbf{R}: \alpha^{\prime}(\xi) \neq 0\right\} \subseteq\left[0, \theta_{c}\right], \\
\left|\alpha^{\prime \prime}(\xi)\right| \leq c_{\alpha} \quad \forall \xi \in \mathbf{R},
\end{array}
$$

for $c_{\alpha}>0$. Let us point out that (3.27) implies in particular that

$$
\left|\alpha^{\prime}(\xi)\right| \leq c_{\alpha} \theta_{c}, \quad\left|\xi \alpha^{\prime}(\xi)\right| \leq c_{\alpha} \theta_{c}^{2} .
$$

Analogously, to get global existence of a solution for the complete system, we need to restrict the choice of $\lambda$ in (2.5) to the nondecreasing bounded functions, with $\lambda\left(\theta_{*}\right)=0$ and $\lambda$ strictly increasing on a neighborhood of $\theta_{*}$. In addition, we prescribe

$$
\begin{aligned}
& \lambda \in W^{2, \infty}(\mathbf{R}) \cap C^{2}(\mathbf{R}), \\
& \|\lambda\|_{W^{2, \infty}(\mathbf{R})}+\left|\lambda^{\prime}(\xi) \xi\right| \leq \tilde{c}_{\lambda}, \quad\left|\lambda^{\prime \prime}(\xi) \xi\right| \leq c_{\lambda}, \quad \forall \xi \in \mathbf{R},
\end{aligned}
$$

for $\tilde{c}_{\lambda}, c_{\lambda}>0$ (cf. [7]). Note, in particular, the additional boundedness requirements in (3.29) with respect to the natural bound of the functions in $W^{2, \infty}$. Let us point out that the first of the above assumptions (3.27) is justified by the elastic behavior of SMA at high temperature, while the latter (3.29) can be read as a smooth truncation of the standard position (cf. [17])

$$
\lambda(\theta)=\frac{L}{\theta_{*}}\left(\theta-\theta_{*}\right)
$$

where $L>0$ denotes the latent heat associated to the phase transition. However, (3.29) is physically consistent as we are interested in studying the shape memory effect in a neighborhood of the critical phase transition temperature $\theta_{*}$. Indeed, we could retain $\lambda$ linear as in (3.30) near $\theta_{*}$ and then truncate it as required by (3.29). Finally, we should set some compatibility conditions on the involved quantities, but for the sake of clarity, we will specify them later. Nonetheless, as they regard $c_{\alpha}$ and $c_{\lambda}$, which have to 
be smaller than other constants, we can postulate in advance (cf. $[10,8]$ for analogous assumptions)

$$
c_{\alpha} \text { and } c_{\lambda} \text { are sufficiently small. }
$$

From now on, to simplify the notation in (3.3) and (3.4), we let $\delta=\zeta=\eta=k_{0}=1$, as these constants are here assumed to be strictly positive, and include the factor $\frac{1}{2}$ in the subdifferential operator.

REMARK 3.1. We have to remark at once that in order to prove that the above model admits existence and uniqueness of the solution, we are not able to deal with a strong version of (3.3)-(3.5). In particular, we cannot deal with the natural $H^{2} \times H^{2}$ extension of the subdifferential $\partial I_{\widetilde{K}}$, i.e., the subdifferential $\partial I_{K}$ of the convex $K \subseteq H^{2}$. Hence, we are led to impose the internal constraint $\left(\chi_{1}, \chi_{2}\right) \in \widetilde{K}$ in the $\left(V^{\prime}\right)^{2} \times V^{2}$ framework. We consider the convex subset of $V^{2}$ given by $K \cap V^{2}$ (where $K$ has been introduced in $(3.17)$ ), and denote by $\partial I_{K, V}$ the maximal monotone operator corresponding to the subdifferential $(V)^{2} \rightarrow\left(V^{\prime}\right)^{2}$ of the indicator function $I_{K \cap V}$ of $K \cap V^{2}$, namely (cf. [3])

$$
\begin{aligned}
& \left(\xi_{1}, \xi_{2}\right) \in\left(V^{\prime}\right)^{2} \text { belongs to } \partial I_{K, V}\left(\chi_{1}, \chi_{2}\right) \text { if and only if } \\
& \left(\chi_{1}, \chi_{2}\right) \in K \cap V^{2} \text { and } \sum_{i=1}^{2}\left\langle\xi_{i}, \gamma_{i}-\chi_{i}\right\rangle \leq 0, \quad \forall\left(\gamma_{1}, \gamma_{2}\right) \in K \cap V^{2} .
\end{aligned}
$$

Finally, concerning the regularity of the data, we prescribe that

$$
\begin{aligned}
& r \in L^{2}\left(0, T ; L^{2}(\Omega)\right), \\
& h \in W^{1,1}\left(0, T ; L^{2}(\Gamma)\right), \\
& \mathbf{G} \in H^{1}\left(0, T ; L^{2}(\Omega)^{3}\right), \\
& \mathbf{g} \in H^{1}\left(0, T ; L^{2}\left(\Gamma_{1}\right)^{3}\right),
\end{aligned}
$$

and introduce two functions $\mathcal{R}$ and $\mathcal{G}$ specified by

$$
\begin{aligned}
& \langle\mathcal{R}, v\rangle=\left\langle\mathcal{R}_{1}, v\right\rangle+\left\langle\mathcal{R}_{2}, v\right\rangle=\int_{\Omega} r v+\int_{\Gamma} h v_{\mid \Gamma} \quad v \in V, \\
& \mathbf{w}^{\prime}\langle\mathcal{G}, \mathbf{v}\rangle_{\mathbf{W}}=\int_{\Omega} \mathbf{G} \cdot \mathbf{v}+\int_{\Gamma_{1}} \mathbf{g} \cdot \mathbf{v}_{\mid \Gamma} \quad \mathbf{v} \in \mathbf{W},
\end{aligned}
$$

so that, by $(3.33)-(3.36)$, it is natural to postulate

$$
\begin{aligned}
& \mathcal{R}=\mathcal{R}_{1}+\mathcal{R}_{2} \in L^{2}(0, T ; H)+W^{1,1}\left(0, T ; V^{\prime}\right), \\
& \mathcal{G} \in H^{1}\left(0, T ; \mathbf{W}^{\prime}\right) .
\end{aligned}
$$

Here is a precise formulation of the problem we are dealing with (cf. (3.3)-(3.5), (2.49), (3.6), (3.8), (2.51)-(2.53), and (2.55)).

Problem $P$. Find $\theta, \mathbf{u}, \chi_{1}$, and $\chi_{2}$ satisfying

$$
\begin{aligned}
& \theta(0)=\theta_{0}, \\
& \chi_{i}(0)=\chi_{i 0} \quad i=1,2,
\end{aligned}
$$


and fulfilling a.e. in $(0, T)$

$$
\begin{aligned}
& F\left(\theta, \mathbf{u}, \chi_{1}, \chi_{2}\right) \theta_{t}+A \theta=\mathcal{R}+\theta \lambda^{\prime}(\theta) \chi_{1 t}+\theta \alpha^{\prime}(\theta) \operatorname{div} \mathbf{u} \chi_{2 t} \\
& +\theta \alpha^{\prime}(\theta) \chi_{2} \operatorname{div} \mathbf{u}_{t} \quad \text { in } V^{\prime} \\
& \left(\begin{array}{l}
\chi_{1 t} \\
\chi_{2 t}
\end{array}\right)+\left(\begin{array}{l}
A \chi_{1 t} \\
A \chi_{2 t}
\end{array}\right)+\left(\begin{array}{l}
A \chi_{1} \\
A \chi_{2}
\end{array}\right)+\left(\begin{array}{l}
\xi_{1} \\
\xi_{2}
\end{array}\right)=\left(\begin{array}{c}
-\lambda(\theta) \\
-\alpha(\theta) \operatorname{div} \mathbf{u}
\end{array}\right) \quad \text { in }\left(V^{\prime}\right)^{2}, \\
& \text { for some }\left(\begin{array}{l}
\xi_{1} \\
\xi_{2}
\end{array}\right) \in \partial I_{K, V}\left(\chi_{1}, \chi_{2}\right) \\
& \mathcal{H} \mathbf{u}+\mathcal{B}\left(\alpha(\theta) \chi_{2}\right)=\mathcal{G} \quad \text { in } \mathbf{W}^{\prime}
\end{aligned}
$$

where $F$ in (3.43) is specified by

$$
F\left(\theta, \mathbf{u}, \chi_{1}, \chi_{2}\right)=c_{s}-\theta \alpha^{\prime \prime}(\theta) \operatorname{div} \mathbf{u} \chi_{2}+\theta \lambda^{\prime \prime}(\theta)\left(1-\chi_{1}\right) .
$$

REMARK 3.2. Notice that the variational inclusion (3.44) governing the dynamics of the phases is written in the abstract setting of the $\left(V^{\prime}\right)^{2}-V^{2}$ duality pairing. Nonetheless, even if it cannot be written a.e. in $Q$, it retains its physical consistence since it forces the phases to attain only meaningful values. Indeed, the abstract relation $\left(\xi_{1}, \xi_{2}\right) \in$ $\partial I_{K, V}\left(\chi_{1}, \chi_{2}\right)$ means (cf. (3.32)) that $\left(\chi_{1}, \chi_{2}\right) \in K \cap V^{2}$ and consequently (cf. (3.17)) $\left(\chi_{1}, \chi_{2}\right) \in \widetilde{K}$ a.e. in $Q$.

REMARK 3.3. We point out that since in our case $I_{\widetilde{K}}: \mathbf{R}^{2} \rightarrow[0,+\infty]$ is a proper, convex, lower semicontinuous function, whenever $\left(\xi_{1}, \xi_{2}\right) \in \partial I_{K, V}\left(\chi_{1}, \chi_{2}\right)$ and $\left(\xi_{1}, \xi_{2}\right) \in$ $H^{2}$ a.e. in $(0, T)$ we have $\left(\xi_{1}, \xi_{2}\right) \in \partial I_{K}\left(\chi_{1}, \chi_{2}\right)$ a.e. in $(0, T)$. In particular, in this situation we would be allowed to deduce also that $\left(\xi_{1}, \xi_{2}\right) \in \partial I_{\widetilde{K}}\left(\chi_{1}, \chi_{2}\right)$ a.e. in $Q$. Indeed, by the result proved by [4, Proposition 2.5], we can infer that $\partial I_{K}=H^{2} \cap \partial I_{K, V}$. Nonetheless, for the sake of completeness, we should observe that this last relation cannot be extended to general functionals, as the reader can verify by referring to the example presented in [4].

Theorem 3.1. Let (3.25)-(3.26), (3.27), (3.29), (3.31), and (3.39)-(3.40) hold. Then, there exists a quadruple of functions $\left(\theta, \mathbf{u}, \chi_{1}, \chi_{2}\right)$ solving the Problem $\mathrm{P}$ and fulfilling

$$
\begin{aligned}
& \theta \in H^{1}(0, T ; H) \cap L^{\infty}(0, T ; V), \\
& \mathbf{u} \in H^{1}(0, T ; \mathbf{W}) \quad \text { with } \operatorname{div} \mathbf{u} \in H^{1}\left(0, T ; H^{2}(\Omega)\right) \\
& \chi_{i} \in W^{1, \infty}(0, T ; V) \cap L^{\infty}\left(0, T ; H^{2}(\Omega)\right), \quad i=1,2 .
\end{aligned}
$$

In particular, there exists a positive constant $c_{1}$ depending only on $C, c_{K},\|\alpha\|_{L^{\infty}(\mathbf{R})}$, $\|\mathcal{G}\|_{L^{\infty}\left(0, T ; \mathbf{W}^{\prime}\right)}$ and $\Omega$, such that

$$
\|\operatorname{div} \mathbf{u}\|_{L^{\infty}(Q)} \leq c_{1} .
$$

REMARK 3.4. We omit the proof of the above statement for which we refer to a subsequent paper where the existence result will be extended to a system where some quadratic dissipative terms are retained in the energy balance. On the other hand, we point out that in this latter case a uniqueness result seems very difficult to recover, since the highly nonlinear quadratic terms in the energy balance allow us to estimate the difference of two values for the temperature only in terms of the difference of the 
phases' time derivatives, which seems difficult to be treated only by using (3.44), due to the presence of the subdifferential operator. On the contrary, for the solutions of $P$ fulfilling (3.47)-(3.49) we can state a uniqueness result, which is presented in Theorem 3.2 .

Notice that by virtue of (3.50) we can now make precise the assumption (3.31) as follows:

$$
\begin{gathered}
c_{2}:=c_{s}-c_{\lambda}\left(1+c_{K}\right)-\theta_{c} c_{a} c_{1} c_{K}>0, \\
\left(\theta_{c} c_{\alpha} c_{K}\left(\theta_{c}+1\right)\right)^{2} \leq c_{2}\left(\lambda_{L}+2 \mu_{L} / 3\right) .
\end{gathered}
$$

In particular, let us point out that the first of the above assumptions (3.51) corresponds to require that the specific heat of the phase transition, which is represented by the coefficient $F$ of $\theta_{t}$ in the energy balance, is a.e. positive, as the reader can easily verify after observing that due to (3.18), (3.27), (3.29), and (3.50), there holds (cf. (3.46))

$$
F\left(\theta, \mathbf{u}, \chi_{1}, \chi_{2}\right) \geq c_{s}-c_{\lambda}\left(1+c_{K}\right)-\theta_{c} c_{\alpha} c_{1} c_{K} \quad \text { a.e. in } Q .
$$

Hence, the following uniqueness theorem holds.

Theorem 3.2. Let $\left(\theta, \mathbf{u}, \chi_{1}, \chi_{2}\right)$ be a solution of the problem $P$ fulfilling $(3.47)-(3.49)$ (and (3.50)), and assume that (3.27), (3.29), and (3.31) (i.e. (3.51)-(3.52)) hold. Then, this solution is unique.

4. Proof of Theorem 3.2. In this section, we aim to prove the uniqueness result stated by Theorem 3.2 ; i.e., we show uniqueness of the solution to the system (3.43)(3.45) with the regularity specified by (3.47)-(3.49) and fulfilling (3.50). First, let us make some remarks about useful notations we will use in the following. We assume that the problem $P$ admits two solutions

$$
\mathcal{S}_{1}=\left\{\theta_{1}, \mathbf{u}_{1}, \chi_{11}, \chi_{21}\right\} \quad \text { and } \mathcal{S}_{2}=\left\{\theta_{2}, \mathbf{u}_{2}, \chi_{12}, \chi_{22}\right\},
$$

with regularity (3.47)-(3.49), (3.50), and fulfilling the Cauchy conditions (3.41)-(3.42). In the sequel, we will denote by $\bar{f}$ the difference of two functions $f_{1}$ and $f_{2}$, i.e.,

$$
\bar{f}=f_{1}-f_{2}
$$

and make use of two trivial identities

$$
a_{1} b_{1}-a_{2} b_{2}=\overline{a b}=\bar{a} b_{2}+a_{1} \bar{b}=\bar{a} b_{1}+a_{2} \bar{b}
$$

so that, without loss of generality, we can rewrite (4.3) and subsequent computations omitting subscripts, i.e.,

$$
\overline{a b}=\bar{a} b+a \bar{b} .
$$

We first write (3.43) in the following equivalent way:

$$
\begin{aligned}
& c_{s} \theta_{t}+\left(\left(\alpha(\theta)-\theta \alpha^{\prime}(\theta)\right) \chi_{2} \operatorname{div} \mathbf{u}\right)_{t}+\left(\left(\theta \lambda^{\prime}(\theta)-\lambda(\theta)\right)\left(1-\chi_{1}\right)\right)_{t}+A \theta \\
& =\mathcal{R}+\alpha(\theta) \chi_{2} \operatorname{div} \mathbf{u}_{t}+\alpha(\theta) \operatorname{div} \mathbf{u} \chi_{2 t}+\lambda(\theta) \chi_{1 t} .
\end{aligned}
$$


Hence, we take the difference of (4.5), written for $\mathcal{S}_{1}$ and $\mathcal{S}_{2}$, and integrate it in time:

$$
\begin{aligned}
& c_{s} \bar{\theta}+\overline{\left(\alpha(\theta)-\theta \alpha^{\prime}(\theta)\right) \chi_{2} \operatorname{div} \mathbf{u}}+\overline{\left(\theta \lambda^{\prime}(\theta)-\lambda(\theta)\right)\left(1-\chi_{1}\right)} \\
& +1 * \overline{A \bar{\theta}=1} * \overline{\alpha(\theta) \chi_{2 t} \operatorname{div} \mathbf{u}}+1 * \overline{\alpha(\theta) \chi_{2} \operatorname{div} \mathbf{u}_{t}} \\
& +1 * \overline{\lambda(\theta) \chi_{1 t}},
\end{aligned}
$$

where by $*$ we denote the usual convolution product over the interval $(0, t)$, namely

$$
(a * b)(t)=\int_{0}^{t} a(t-s) b(s) d s .
$$

Hence, by use of the equality (4.4) and exploiting some integrations by parts, from (4.6) we get

$$
\begin{aligned}
& c_{s} \bar{\theta}+\left(\overline{\alpha(\theta)-\theta \alpha^{\prime}(\theta)}\right) \chi_{2} \operatorname{div} \mathbf{u}+\left(\overline{\theta \lambda^{\prime}(\theta)-\lambda(\theta)}\right)\left(1-\chi_{1}\right)+1 * A \bar{\theta} \\
& =-\left(\alpha(\theta)-\theta \alpha^{\prime}(\theta)\right) \overline{\chi_{2}} \operatorname{div} \mathbf{u}-\left(\alpha(\theta)-\theta \alpha^{\prime}(\theta)\right) \chi_{2} \operatorname{div} \overline{\mathbf{u}}+\left(\theta \lambda^{\prime}(\theta)-\lambda(\theta)\right) \overline{\chi_{1}} \\
& +1 * \overline{\alpha(\theta) \operatorname{div} \mathbf{u}} \chi_{2 t}+1 * \alpha(\theta) \operatorname{div} \mathbf{u} \overline{\chi_{2 t}}+1 * \overline{\alpha(\theta) \chi_{2}} \operatorname{div} \mathbf{u}_{t} \\
& +1 * \alpha(\theta) \chi_{2} \operatorname{div} \overline{\mathbf{u}}_{t}+1 * \overline{\lambda(\theta)} \chi_{1 t}+1 * \lambda(\theta) \overline{\chi_{1 t}} \\
& =\theta \alpha^{\prime}(\theta) \operatorname{div} \mathbf{u} \overline{\chi_{2}}+\theta \alpha^{\prime}(\theta) \chi_{2} \operatorname{div} \overline{\mathbf{u}}+\theta \lambda^{\prime}(\theta) \overline{\chi_{1}} \\
& +1 * \overline{\alpha(\theta) \operatorname{div} \mathbf{u}} \chi_{2 t}-1 *(\alpha(\theta) \operatorname{div} \mathbf{u})_{t} \overline{\chi_{2}}+1 * \overline{\alpha(\theta) \chi_{2}} \operatorname{div} \mathbf{u}_{t} \\
& -1 *\left(\alpha(\theta) \chi_{2}\right)_{t} \operatorname{div} \overline{\mathbf{u}}+1 * \overline{\lambda(\theta)} \chi_{1 t}-1 * \lambda(\theta)_{t} \overline{\chi_{1}} \text {. }
\end{aligned}
$$

Then, we can test (4.8) by $\bar{\theta}$ and integrate in time over $(0, t)$, with $t$ arbitrary in $(0, T)$. Owing to (3.27) and (3.29) we first observe that $\left(\alpha(\theta)-\theta \alpha^{\prime}(\theta)\right)$ and $\left(\lambda(\theta)-\theta \lambda^{\prime}(\theta)\right)$ are Lipschitz. Hence, let us point out that

$$
\left(\alpha(\theta)-\theta \alpha^{\prime}(\theta)\right)^{\prime}=-\alpha^{\prime \prime}(\theta) \theta, \quad\left(\theta \lambda^{\prime}(\theta)\right)^{\prime}=\theta \lambda^{\prime \prime}(\theta)
$$

from which we can infer that (cf. (3.27))

$$
\overline{\mid \alpha(\theta)-\theta \alpha^{\prime}(\theta)}\left|\leq \theta_{c} c_{\alpha}\right| \bar{\theta} \mid \quad \text { a.e. in } Q,
$$

and analogously (cf. (3.29))

$$
\left|\overline{\theta \lambda^{\prime}(\theta)-\lambda(\theta)}\right| \leq c_{\lambda}|\bar{\theta}| \quad \text { a.e. in } Q .
$$

Thus, we can write

$$
\begin{aligned}
& \int_{0}^{t} \int_{\Omega}\left(\left|\overline{\alpha(\theta)-\theta \alpha^{\prime}(\theta)}\right| \chi_{2} \operatorname{div} \mathbf{u}+\left|\overline{\theta \lambda^{\prime}(\theta)-\lambda(\theta)}\right|\left(1-\chi_{2}\right)\right)|\bar{\theta}| \\
& \quad \leq\left(\theta_{c} c_{\alpha} c_{K} c_{1}+c_{\lambda}\left(1+c_{K}\right)\right)\|\bar{\theta}\|_{L^{2}(0, t ; H)}^{2},
\end{aligned}
$$

so that, owing to (3.51) (cf. (3.53)), and integrating by parts in time, we can write

$$
c_{2}\|\bar{\theta}\|_{L^{2}(0, t ; H)}^{2}+\frac{1}{2}\|1 * \nabla \bar{\theta}(t)\|_{H}^{2} \leq \sum_{j=1}^{9} I_{j}(t),
$$


where the integrals $I_{j}(t), j=1, \ldots, 9$, are specified as follows:

$$
\begin{aligned}
& I_{1}(t)=\int_{0}^{t} \int_{\Omega} \theta \alpha^{\prime}(\theta) \operatorname{div} \mathbf{u} \overline{\chi_{2}} \bar{\theta} \\
& I_{2}(t)=\int_{0}^{t} \int_{\Omega} \theta \alpha^{\prime}(\theta) \chi_{2} \operatorname{div} \overline{\mathbf{u}} \bar{\theta} \\
& I_{3}(t)=\int_{0}^{t} \int_{\Omega} \theta \lambda^{\prime}(\theta) \overline{\chi_{1}} \bar{\theta} \\
& I_{4}(t)=\int_{0}^{t} \int_{\Omega}\left(1 * \overline{\left.\alpha(\theta) \operatorname{div} \mathbf{u} \chi_{2 t}\right) \bar{\theta}}\right. \\
& I_{5}(t)=-\int_{0}^{t} \int_{\Omega}\left(1 *(\alpha(\theta) \operatorname{div} \mathbf{u})_{t} \overline{\chi_{2}}\right) \bar{\theta} \\
& I_{6}(t)=\int_{0}^{t} \int_{\Omega}\left(1 * \overline{\alpha(\theta) \chi_{2}} \operatorname{div} \mathbf{u}_{t}\right) \bar{\theta} \\
& I_{7}(t)=-\int_{0}^{t} \int_{\Omega}\left(1 *\left(\alpha(\theta) \chi_{2}\right)_{t} \operatorname{div} \overline{\mathbf{u}}\right) \bar{\theta} \\
& I_{8}(t)=\int_{0}^{t} \int_{\Omega}\left(1 * \overline{\lambda(\theta)} \chi_{1 t}\right) \bar{\theta} \\
& I_{9}(t)=-\int_{0}^{t} \int_{\Omega}\left(1 * \lambda(\theta)_{t} \overline{\chi_{1}}\right) \bar{\theta}
\end{aligned}
$$

and they will be handled in a moment.

We first treat the integrals $I_{1}, I_{2}$, and $I_{3}$ by following analogous procedures. By $(3.28)$ and (3.50), just by use of Hölder inequality, we get

$$
\begin{aligned}
\left|I_{1}(t)\right| & \leq \theta_{c}^{2} c_{\alpha} c_{1}\left\|\overline{\chi_{2}}\right\|_{L^{2}(0, t ; H)}\|\bar{\theta}\|_{L^{2}(0, t ; H)} \\
& \leq \frac{c_{2}}{32}\|\bar{\theta}\|_{L^{2}(0, t ; H)}^{2}+c\left\|\overline{\chi_{2}}\right\|_{L^{2}(0, t ; H)}^{2} .
\end{aligned}
$$

Let us point out that here and in the sequel we denote by $c$ possibly different positive constants which do not depend on the difference of the two solutions $\mathcal{S}_{1}$ and $\mathcal{S}_{2}$. Analogously, on account also of (3.18), we can treat the other integrals as follows:

$$
\left|I_{2}(t)\right| \leq \theta_{c}^{2} c_{\alpha} c_{K}\|\bar{\theta}\|_{L^{2}(0, t ; H)}\|\operatorname{div} \overline{\mathbf{u}}\|_{L^{2}(0, t ; H)},
$$

and, due to $(3.29)$,

$$
\begin{aligned}
\left|I_{3}(t)\right| & \leq \tilde{c}_{\lambda}\left\|\overline{\chi_{1}}\right\|_{L^{2}(0, t ; H)}\|\bar{\theta}\|_{L^{2}(0, t ; H)} \\
& \leq \frac{c_{2}}{32}\|\bar{\theta}\|_{L^{2}(0, t ; H)}^{2}+c\left\|\overline{\chi_{1}}\right\|_{L^{2}(0, t ; H)}^{2} .
\end{aligned}
$$

Now, we integrate by parts in time $I_{4}$, and, by use of a generalized version of the Hölder inequality and the continuous embedding $H^{1}(\Omega) \hookrightarrow L^{4}(\Omega)$, we get (cf. also (3.49) and $(3.13))$ 


$$
\begin{aligned}
\left|I_{4}(t)\right| \leq & \int_{\Omega}|(1 * \bar{\theta})(t)| \int_{0}^{t}\left|\overline{\alpha(\theta) \operatorname{div} \mathbf{u}} \chi_{2 t}\right|+\int_{0}^{t} \int_{\Omega}\left|1 * \bar{\theta} \| \overline{\alpha(\theta) \operatorname{div} \mathbf{u}} \chi_{2 t}\right| \\
\leq & \|(1 * \bar{\theta})(t)\|_{L^{4}(\Omega)} \int_{0}^{t}\left\|\chi_{2 t}\right\|_{L^{4}(\Omega)}\left(c_{1} \theta_{c} c_{\alpha}\|\bar{\theta}\|_{H}+\|\alpha\|_{L^{\infty}(\mathbf{R})}\|\operatorname{div} \overline{\mathbf{u}}\|_{H}\right) \\
& \quad+\int_{0}^{t}\|1 * \bar{\theta}\|_{L^{4}(\Omega)}\left\|\chi_{2 t}\right\|_{L^{4}(\Omega)}\left(c_{1} \theta_{c} c_{\alpha}\|\bar{\theta}\|_{H}+\|\alpha\|_{L^{\infty}(\mathbf{R})}\|\operatorname{div} \overline{\mathbf{u}}\|_{H}\right) \\
\leq & \frac{c_{9}}{10}\|1 * \bar{\theta}\|_{L^{\infty}(0, t ; V)}^{2}+c_{5}\left(\int_{0}^{t}\left\|\chi_{2 t}\right\|_{V}^{2}\right)\left(\|\bar{\theta}\|_{L^{2}(0, t ; H)}^{2}+\|\overline{\mathbf{u}}\|_{L^{2}(0, t ; \mathbf{W})}^{2}\right)
\end{aligned}
$$

for a positive constant $c_{9}$ we will fix later. Analogously, we can infer that

$$
\begin{aligned}
\left|I_{5}(t)\right| \leq \int_{\Omega}\left|\left(1 *(\alpha(\theta) \operatorname{div} \mathbf{u})_{t} \overline{\chi_{2}}\right)(t)\right||(1 * \bar{\theta})(t)| \\
\quad+\int_{0}^{t} \int_{\Omega}\left|(\alpha(\theta) \operatorname{div} \mathbf{u})_{t} \overline{\chi_{2}}\right||1 * \bar{\theta}| \\
\leq\|(1 * \bar{\theta})(t)\|_{L^{4}(\Omega)} \int_{0}^{t}\left\|(\alpha(\theta) \operatorname{div} \mathbf{u})_{t}\right\|_{H}\left\|\overline{\chi_{2}}\right\|_{L^{4}(\Omega)} \\
\quad+\int_{0}^{t}\|1 * \bar{\theta}\|_{L^{4}(\Omega)}\left\|(\alpha(\theta) \operatorname{div} \mathbf{u})_{t}\right\|_{H}\left\|\overline{\chi_{2}}\right\|_{L^{4}(\Omega)} \\
\leq \frac{c_{9}}{10}\|1 * \bar{\theta}\|_{L^{\infty}(0, t ; V)}^{2}+c\left\|\overline{\chi_{2}}\right\|_{L^{2}(0, t ; V)}^{2},
\end{aligned}
$$

where in particular the constant $c$ depends on $\left\|(\alpha(\theta) \operatorname{div} \mathbf{u})_{t}\right\|_{L^{2}(0, t ; H)}$ (cf. (3.47), (3.48), and (3.27)). Hence, we can apply once more the Hölder inequality on account of (3.48), (3.27), and (3.18), exploit the Sobolev embedding $H^{2}(\Omega) \hookrightarrow L^{\infty}(\Omega)$, and finally write (cf. also [8] for a similar estimate)

$$
\begin{aligned}
\left|I_{6}(t)\right| & \leq \int_{0}^{t} \int_{\Omega}|\bar{\theta}|\left|1 *\left(\overline{\alpha(\theta)} \chi_{2}+\alpha(\theta) \overline{\chi_{2}}\right) \operatorname{div} \mathbf{u}_{t}\right| \\
& \leq \int_{0}^{t}\|\bar{\theta}\|_{H} \int_{0}^{\tau}\left\|\operatorname{div} \mathbf{u}_{t}\right\|_{L^{\infty}(\Omega)}\left(\theta_{c} c_{\alpha} c_{K}\|\bar{\theta}\|_{H}+\|\alpha\|_{L^{\infty}(\mathbf{R})}\left\|\overline{\chi_{2}}\right\|_{H}\right) \\
& \leq \frac{c_{2}}{32}\|\bar{\theta}\|_{L^{2}(0, t ; H)}^{2}+c_{6}\left(\int_{0}^{t}\left\|\operatorname{div} \mathbf{u}_{t}\right\|_{H^{2}(\Omega)}^{2}\right)\left(\left\|\overline{\chi_{2}}\right\|_{L^{2}(0, t ; H)}^{2}+\|\bar{\theta}\|_{L^{2}(0, t ; H)}^{2}\right) .
\end{aligned}
$$


Analogously, we can integrate by parts $I_{7}$ and owing to (3.27), (3.47), and (3.49), we are allowed to deduce

$$
\begin{aligned}
\left|I_{7}(t)\right| \leq & \int_{\Omega}\left|\left(1 *\left(\alpha(\theta) \chi_{2}\right)_{t} \operatorname{div} \overline{\mathbf{u}}\right)(t)\right||(1 * \bar{\theta})(t)| \\
& \quad+\int_{0}^{t} \int_{\Omega}\left|\left(\alpha(\theta) \chi_{2}\right)_{t} \operatorname{div} \overline{\mathbf{u}}\right||1 * \bar{\theta}| \\
\leq & \|(1 * \bar{\theta})(t)\|_{L^{4}(\Omega)} \int_{0}^{t}\|\operatorname{div} \overline{\mathbf{u}}\|_{L^{4}(\Omega)}\left\|\left(\alpha(\theta) \chi_{2}\right)_{t}\right\|_{H} \\
& \quad+\int_{0}^{t}\|1 * \bar{\theta}\|_{L^{4}(\Omega)}\|\operatorname{div} \overline{\mathbf{u}}\|_{L^{4}(\Omega)}\left\|\left(\alpha(\theta) \chi_{2}\right)_{t}\right\|_{H} \\
\leq & \frac{c_{9}}{10}\|1 * \bar{\theta}\|_{L^{\infty}(0 . t ; V)}^{2} \\
& +c_{7}\left(\int_{0}^{t}\left\|\left(\alpha(\theta) \chi_{2}\right)_{t}\right\|_{H}^{2}\right)\|\overline{\mathbf{u}}\|_{L^{2}(0, t ; \mathbf{W})}^{2} .
\end{aligned}
$$

Next, by a similar procedure as that we have exploited to handle $I_{4}$, and owing to (3.29) and (3.49), it is now a standard matter to deduce

$$
\begin{aligned}
\left|I_{8}(t)\right| & \leq \int_{\Omega}\left|\left(1 * \overline{\lambda(\theta)} \chi_{1 t}\right)(t)\right||(1 * \bar{\theta})(t)|+\int_{0}^{t} \int_{\Omega}\left|\overline{\lambda(\theta)} \chi_{1 t}\right||1 * \bar{\theta}| \\
& \leq \frac{c_{9}}{10}\|1 * \bar{\theta}\|_{L^{\infty}(0, t ; V)}^{2}+c_{8}\left(\int_{0}^{t}\left\|\chi_{1 t}\right\|_{V}^{2}\right)\|\bar{\theta}\|_{L^{2}(0, t ; H)}^{2} .
\end{aligned}
$$

Finally, we deal with the last integral

$$
\begin{aligned}
\left|I_{9}(t)\right| \leq & \int_{\Omega}\left|\left(1 * \lambda(\theta)_{t} \overline{\chi_{1}}\right)(t)\right||(1 * \bar{\theta})(t)| \\
& \quad+\int_{0}^{t} \int_{\Omega}\left|\lambda(\theta)_{t} \overline{\chi_{1}}\right||1 * \bar{\theta}| \\
\leq & \frac{c_{9}}{10}\|1 * \bar{\theta}\|_{L^{\infty}(0, t ; V)}^{2}+c\left\|\overline{\chi_{1}}\right\|_{L^{2}(0, t: V)}^{2},
\end{aligned}
$$

where $c$ here depends in particular on $\left\|\theta_{t}\right\|_{L^{2}(0, t ; H)}$, as $\lambda(\theta)_{t}=\lambda^{\prime}(\theta) \theta_{t}$ (cf. (3.29) and $(3.47))$.

Now, we can proceed by considering the difference of the equation (3.45) written for $\mathcal{S}_{1}$ and $\mathcal{S}_{2}$, and testing it by $\overline{\mathbf{u}}$. After an integration over $(0, t)$ and owing to (3.15), (3.16), (3.18), and (3.27), we can infer that

$$
\begin{aligned}
& \frac{C}{2}\|\overline{\mathbf{u}}\|_{L^{2}(0, t ; \mathbf{W})}^{2}+\frac{\left(\lambda_{L}+2 \mu_{L} / 3\right)}{2}\|\operatorname{div} \overline{\mathbf{u}}\|_{L^{2}(0, t ; H)}^{2}+\frac{\nu}{2}\|\nabla \operatorname{div} \overline{\mathbf{u}}\|_{L^{2}(0, t ; H)}^{2} \\
& \leq \int_{0}^{t} \int_{\Omega}\left|\overline{\alpha(\theta)} \chi_{2}\right||\operatorname{div} \overline{\mathbf{u}}|+\left|\alpha(\theta) \overline{\chi_{2}}\right||\operatorname{div} \overline{\mathbf{u}}| \\
& \leq \theta_{c} c_{\alpha} c_{K}\|\bar{\theta}\|_{L^{2}(0 . t: H)}\|\operatorname{div} \overline{\mathbf{u}}\|_{L^{2}(0 . t ; H)} \\
&+3^{1 / 2}\|\alpha\|_{L^{\infty}(\mathbf{R})}\left\|\overline{\chi_{2}}\right\|_{L^{2}(0, t ; H)}\|\overline{\mathbf{u}}\|_{L^{2}(0, t ; \mathbf{W})} \\
& \leq \theta_{c} c_{\alpha} c_{K}\|\bar{\theta}\|_{L^{2}(0, t ; H)}\|\operatorname{div} \overline{\mathbf{u}}\|_{L^{2}(0, t ; H)} \\
&+\frac{C}{4}\|\overline{\mathbf{u}}\|_{L^{2}(0 . t ; \mathbf{W})}^{2}+c\left\|\overline{\chi_{2}}\right\|_{L^{2}(0, t ; H)}^{2}
\end{aligned}
$$


Finally, we write (3.44) for $\mathcal{S}_{1}$ and $\mathcal{S}_{2}$, take the difference, test it by $\left(\overline{\chi_{1}}, \overline{\chi_{2}}\right)$, and integrate over $(0, t)$. Let us first note that, by monotonicity of the operator $\partial I_{K, V}$, we have

$$
\sum_{i=1}^{2} \int_{0}^{t}\left\langle\xi_{i 1}-\xi_{i 2}, \chi_{i 1}-\chi_{i 2}\right\rangle \geq 0
$$

since $\left(\xi_{1 j}, \xi_{2 j}\right) \in \partial I_{K, V}\left(\chi_{1 j}, \chi_{2 j}\right)$. Thus, by integrating by parts in time and recalling (3.27), (3.29), and (3.50), it is straightforward to deduce

$$
\begin{aligned}
& \sum_{j=1}^{2} \frac{1}{2}\left\|\overline{\chi_{j}}(t)\right\|_{V}^{2}+\left\|\nabla \overline{\chi_{j}}\right\|_{L^{2}(0, t ; H)}^{2} \leq \tilde{c}_{\lambda}\|\bar{\theta}\|_{L^{2}(0, t ; H)}\left\|\overline{\chi_{1}}\right\|_{L^{2}(0, t ; H)} \\
& \quad+\left(\theta_{c} c_{\alpha} c_{1}\|\bar{\theta}\|_{L^{2}(0, t ; H)}+\|\alpha\|_{L^{\infty}(\mathbf{R})}\|\operatorname{div} \overline{\mathbf{u}}\|_{L^{2}(0, t ; H)}\right)\left\|\overline{\chi_{2}}\right\|_{L^{2}(0, t ; H)} \\
& \leq \frac{c_{2}}{32}\|\bar{\theta}\|_{L^{2}(0, t ; H)}^{2}+\frac{C}{16}\|\overline{\mathbf{u}}\|_{L^{2}(0, t ; \mathbf{W})}^{2}+c \sum_{j=1}^{2}\left\|\overline{\chi_{j}}\right\|_{L^{2}(0, t ; H)}^{2}
\end{aligned}
$$

Now, we combine (4.22)-(4.30) with (4.12) and add the resulting inequality to (4.31) and (4.33). In particular, after recalling that

$$
\|1 * \bar{\theta}(t)\|_{H} \leq T^{1 / 2}\|\bar{\theta}\|_{L^{2}(0, t ; H)},
$$

we can take, i.e.,

$$
c_{9}=\min \left\{\frac{1}{2}, \frac{c_{2}}{8 T}\right\}
$$

and write

$$
\begin{aligned}
& \frac{3 c_{2}}{4}\|\bar{\theta}\|_{L^{2}(0, t ; H)}^{2}+c_{9}\|(1 * \bar{\theta})(t)\|_{V}^{2}+\frac{3 C}{16}\|\overline{\mathbf{u}}\|_{L^{2}(0, t ; \mathbf{W})}^{2} \\
& \quad+\frac{\left(\lambda_{L}+2 \mu_{L} / 3\right.}{2}\|\operatorname{div} \overline{\mathbf{u}}\|_{L^{2}(0, t ; H)}^{2} \\
& \quad+\sum_{j=1}^{2} \frac{1}{2}\left\|\overline{\chi_{j}}(t)\right\|_{V}^{2} \leq \frac{c_{9}}{2}\|1 * \bar{\theta}\|_{L^{\infty}(0, t ; V)}^{2}+c \sum_{j=1}^{2}\left\|\overline{\chi_{j}}\right\|_{L^{2}(0, t ; V)}^{2} \\
& \quad+\theta_{c} c_{\alpha} c_{K}\left(1+\theta_{c}\right)\|\bar{\theta}\|_{L^{2}(0, t ; H)}\|\operatorname{div} \overline{\mathbf{u}}\|_{L^{2}(0, t ; H)} \\
& \quad+c_{5}\left(\int_{0}^{t}\left\|\chi_{2 t}\right\|_{V}^{2}\right)\left(\|\bar{\theta}\|_{L^{2}(0, t ; H)}^{2}+\|\overline{\mathbf{u}}\|_{L^{2}(0, t ; \mathbf{W})}^{2}\right. \\
& \quad+c_{6}\left(\int_{0}^{t}\left\|\operatorname{div} \mathbf{u}_{t}\right\|_{H^{2}(\Omega)}^{2}\right)\|\bar{\theta}\|_{L^{2}(0, t ; H)}^{2}+c_{7}\left(\int_{0}^{t}\left\|\left(\alpha(\theta) \chi_{2}\right)_{t}\right\|_{H}^{2}\right)\|\overline{\mathbf{u}}\|_{L^{2}(0, t ; \mathbf{W})}^{2} \\
& \quad+c_{8}\left(\int_{0}^{t}\left\|\chi_{1 t}\right\|_{V}^{2}\right)\|\bar{\theta}\|_{L^{2}(0, t ; H)}^{2} .
\end{aligned}
$$

By recalling (3.52) we can easily verify that

$$
\begin{aligned}
& \theta_{c} c_{\alpha} c_{K}\left(1+\theta_{c}\right)\|\bar{\theta}\|_{L^{2}(0, t ; H)}\|\operatorname{div} \overline{\mathbf{u}}\|_{L^{2}(0, t ; H)} \\
& \quad \leq \frac{c_{2}}{2}\|\bar{\theta}\|_{L^{2}(0, t ; H)}^{2}+\frac{\left(\lambda_{L}+2 \mu_{L} / 3\right)}{2}\|\operatorname{div} \overline{\mathbf{u}}\|_{L^{2}(0, t ; H)}^{2}
\end{aligned}
$$


so that two terms cancel in (4.35). Then, we observe that, due to (3.47)-(3.49), $\left\|\chi_{2 t}\right\|_{V}^{2}$, $\left\|\operatorname{div} \mathbf{u}_{t}\right\|_{H^{2}(\Omega)}^{2},\left\|\left(\alpha(\theta) \chi_{2}\right)_{t}\right\|_{H}^{2}$, and $\left\|\chi_{1 t}\right\|_{V}^{2}$ belong to $L^{1}(0, T)$. Thus, we can find $\hat{t}$ sufficiently small, such that

$$
\begin{aligned}
\max \left\{c_{5} \int_{\tau}^{\hat{t}+\tau}\left\|\chi_{2 t}\right\|_{V}^{2}, c_{6} \int_{\tau}^{\hat{t}+\tau}\left\|\operatorname{div} \mathbf{u}_{t}\right\|_{H^{2}(\Omega)}^{2}\right. \\
\left.c_{7} \int_{\tau}^{\hat{t}+\tau}\left\|\left(\alpha(\theta) \chi_{2}\right)_{t}\right\|_{H}^{2}, c_{8} \int_{\tau}^{\hat{t}+\tau}\left\|\chi_{1 t}\right\|_{V}^{2}\right\} \leq \min \left\{\frac{C}{16}, \frac{c_{2}}{32}\right\},
\end{aligned}
$$

for any $\tau \in[0, T-\hat{t}]$. Thus, at the end, by combining (4.35) with (4.36) and (4.37), we finally get

$$
\begin{aligned}
& \frac{c_{2}}{8}\|\bar{\theta}\|_{L^{2}(0, t ; H)}^{2}+c_{9}\|(1 * \bar{\theta})(t)\|_{V}^{2}+\frac{C}{16}\|\overline{\mathbf{u}}\|_{L^{2}(0, t ; \mathbf{W})}^{2} \\
& \quad+\sum_{j=1}^{2} \frac{1}{2}\left\|\overline{\chi_{j}}(t)\right\|_{V}^{2} \leq c \sum_{j=1}^{2}\left\|\overline{\chi_{j}}\right\|_{L^{2}(0, t ; V)}^{2}+\frac{c_{9}}{2}\|1 * \bar{\theta}\|_{L^{\infty}(0, t ; V)}^{2},
\end{aligned}
$$

i.e., for any $t \in(0, \hat{t})$. Now, by virtue of the generalized version of the Gronwall lemma introduced in [2], from (4.38) it follows

$$
\bar{\theta}=\overline{\chi_{1}}=\overline{\chi_{2}}=\overline{\mathbf{u}}=0 \quad \text { a.e. in } \Omega \times(0, \hat{t}) .
$$

Finally, as we can repeat the same estimates for any interval $(\tau, \tau+\hat{t})$ (cf. (4.37)), by iterating the above procedure we are allowed to extend (4.39) over the whole interval $(0, T)$, which concludes the proof of the uniqueness result.

\section{REFERENCES}

[1] M. Achenback and I. Müller, Model for shape memory, J. Physique, $C_{4}$ Suppl. 12, 12, 163-167 (1982)

[2] C. Baiocchi, Sulle equazioni differenziali astratte del primo e del secondo ordine negli spazi di Hilbert, Ann. Mat. Pura Appl., (4), 76, 233-304 (1967)

[3] V. Barbu, Nonlinear semigroups and differential equations in Banach spaces, Noordhoff, Leyden, 1976

[4] V. Barbu, P. Colli, G. Gilardi, and M. Grasselli, Existence, uniqueness, and long time behavior for a nonlinear Volterra integrodifferential equation, Differential Integral Equations, 13, 1233-1262 (2000)

[5] D. Blanchard, M. Frémond, and A. Visintin, Phase change with dissipation, in: Thermomechanical in coupling solids, H. D. Bui and Q. S. Nguyen eds., North Holland, 1987, 411-418

[6] E. Bonetti, Global solution to a Frémond model for shape memory alloys with thermal memory, Nonlinear Anal., 46, 535-565 (2001)

[7] E. Bonetti, Global solution to a nonlinear phase transition model with dissipation, Adv. Math. Sci. Appl., 12, 355-376 (2002)

[8] N. Chemetov, Uniqueness results for the full Frémond model of shape memory alloys, Z. Anal. Anwendungen, 17, 877-892 (1998)

[9] P. G. Ciarlet, Mathematical Elasticity, Vol. I: Three-Dimensional Elasticity, in: Studies in Mathematics and its Applications, North-Holland, Amsterdam, 1988

[10] P. Colli, Global existence for the three-dimensional Frémond model of shape memory alloys, Nonlinear Anal., 24, 1565-1579, (1995)

[11] P. Colli, M. Frémond, and A. Visintin, Thermo-mechanical evolution of shape memory alloys, Quart. Appl. Math., 48, 31-47 (1990)

[12] P. Colli and J. Sprekels, Positivity of temperature in the general Frémond model for shape memory alloys, Contin. Mech. Thermodyn., 5, 255-264 (1993) 
[13] P. Colli and J. Sprekels, Global solution to the full one-dimensional Frémond model for shape memory alloys, Math. Methods Appl. Sci., 18, 371-385 (1995)

[14] P. Colli and J. Sprekels, Remarks on the existence for the one-dimensional Frémond model of shape memory alloys, Z. Angew. Math. Mech., 76, Suppl. 2, 413-416 (1996)

[15] G. Duvaut and J. L. Lions, Inequalities in mechanics and physics, Springer-Verlag, Berlin-New York, 1976

[16] F. Falk, Elastic phase transitions and nonconvex energy functions, in: "Free Boundary Problems: theory and applications", vol. I-II (K. H. Hoffmann and J. Sprekels, eds.); Pitman Res. Notes Math. Ser. 185, Longman, London, 1990

[17] M. Frémond, Matériaux a mémoire de forme, C. R. Acad. Sci. Paris. Sér. II Méc. Phys. Chim. Sci. Univers. Sci. Terre, 304, 239-244 (1987)

[18] M. Frémond, Shape memory alloys. A thermomechanical model, in "Free Boundary Problems: theory and applications", vol. I-II (K. H. Hoffmann and J. Sprekels, eds.), Pitman Res. Notes Math. Ser. 185, Longman, London, 1990

[19] M. Frémond, Sur l'inégalité de Clausius-Duhem, C. R. Acad. Sci. Sér. II Paris, 311, 757-762 (1990)

[20] M. Frémond, The principle of virtual power in solid mechanics, in: Continuum thermomechanics: the art and the science of modelling material behavior (Paul Germain anniversary vol.), Kluwer Acad. Press, Boston, 2000

[21] M. Frémond, Nonsmooth thermo-mechanics, Springer-Verlag, Heidelberg, 2001

[22] M. Frémond and S. Miyazaki, Shape memory alloys, in: CISM Courses and Lectures No. 351, Springer-Verlag, New York, 1996

[23] M. Frémond and A. Visintin, Dissipation dans le changement de phase. Surfusion. Changement de phase irréversible, C. R. Acad. Paris Sér. II Méc. Phys. Chim. Sci. Univers Sci. Terre, 301, 1265-1268 (1985)

[24] P. Germain, Cours de mécanique des milieux continus, Masson, Paris, 1973

[25] P. Germain, La méthode des puissances en mécanique des milieux continus- ${ }^{0}$ partie, Téorie du second gradient, J. Mécanique, 12, 235-274 (1973)

[26] G. Guénin, Alliages à memoire de forme, Techniques de l'ingénieur, M530, Paris, 1986

[27] J. L. Lions and E. Magenes, Non-homogeneous boundary value problems and applications, Vol. I, Springer-Verlag, Berlin, 1972

[28] J. J. Moreau, Sur les lois de frottement, de viscosité et de plasticité, in: C. R. Acad. Sci., 27, 608-611, Paris, 1970

[29] I. Müller, Pseudoelasticity in shape memory alloys, An extreme case of thermoelasticity, in: Proc. termoelasticità finita, Acc. Naz. dei Lincei, 1985

[30] I. Müller and K. Wilmanski, A model for phase transitions in pseudoelastic bodies, Nuovo Cimento B, 57, 283-318 (1980)

[31] M. Niezgódka and J. Sprekels, Existence of a solution for a mathematical model of structural phase transition in shape memory alloys, Math. Methods Appl. Sci., 10, 197-223 (1988)

[32] E. Patoor and M. Berveiller, Les alliages à mémoire de forme, Hermès, Paris, 1990

[33] N. Shemetov, Existence result for the full one-dimensional Frémond model of shape memory alloys, Adv. Math. Sci. Appl., 8, 157-172 (1998)

[34] J. Sprekels, Shape memory alloys: mathematical models for a class of first order solid-solid phase transitions in metals, Control Cybernet., 19, 287-308 (1990) 